\title{
Characterization and thermal stability of iodinated amorphous conducting carbon films
}

\author{
Latha Kumari $^{* a}$, S.V. Subramanyam ${ }^{\mathrm{a}}$, Arup Gayen ${ }^{\mathrm{b}}$, V. Jayaram ${ }^{\mathrm{b}}$ \\ ${ }^{\mathrm{a}}$ Department of Physics, Indian Institute of Science, Bangalore-560012, India. \\ ${ }^{\mathrm{b}}$ Solid State and Structural Chemistry Unit, Indian Institute of Science, Bangalore- \\ 560012, India
}

\begin{abstract}
In this work, we discuss the structural and transport properties of iodine intercalated amorphous conducting carbon (a-C: I) films prepared by vapor phase pyrolysis of maleic anhydride and iodine. X-ray photoelectron spectroscopy studies reveal the presence of iodine in the a-C: I samples. Microhardness measurements of the films show a decrease in the value of the hardness with an increase in iodine concentration. Iodine incorporation accounts for a change in electrical conductivity of the a-C samples. Magnetoresistance measurements performed on a-C: I samples show a decrease in the value of magnetoresistance. The thermal stability of a-C: I samples has been studied by thermogravimetric analysis, which is found to depend on the host structure and amount of intercalated iodine.
\end{abstract}

Keywords: C. Carbon; E. Electrical properties and measurements; T. Thermal analysis; X. X-ray photoelectron spectroscopy

PACS: 81.05.Uw; 73.50.-h; 81.70.Pg; 79.60.-i

*Corresponding author. Fax: 091-080-360 2602, E-mail address: latha@physics.iisc.ernet.in 


\section{Introduction}

The phenomenon like weak localization, electron-electron interaction, percolation and hopping [1-3] enable a proper understanding of electrical transport in disordered systems. Each of these phenomenon is studied depending on the nature and strength of the disorder in the system. Earlier, the transverse magnetoresistance (MR) measurements were performed on heat-treated soft carbon and hard carbon systems [4]. Mostly in the disordered carbons, magnetoresistance is positive, and increases with the increasing magnetic field and with decreasing temperature. The negative magnetoreistance, which is a typical property for turbostatic carbons, superposes on the positive magnetoresistance [5]. The positive magnetoresistance observed can be attributed to variable-range hopping conduction between localized states in amorphous materials as described by Mott [6].

In our previous work [7], effect of iodine incorporation on the conductivity of the samples in the metallic regimes (samples prepared at $900^{\circ} \mathrm{C}$ and above) has been discussed. a-C: I samples prepared at $900^{\circ} \mathrm{C}$ and above show an anomalous resistivity behavior at low temperatures, a crossover from negative temperature coefficient of resistance (TCR) to positive TCR [7]. Earlier works [8-10] on iodine-intercalated carbon systems depict appreciable change in conductivity.

Magnetoresistance is a sensitive local probe for investigating the scattering process in disordered metallic systems and is closely related to temperature dependence of conductivity. The effect of magnetic field on the weak localization of electrons is similar 
to that of inelastic scattering. It suppresses the phase coherence between the partial waves associated with an electron and this reduces the localization, causing the resistance to decrease with magnetic field (negative magnetoresistance). The contribution to magnetoresistance from electron-electron interaction is positive and is predominantly due to Zeeman splitting of the spin-up and spin-down bands. The magnetoresistance is interpreted to be due to distortion of electron orbit at low fields and due to shrinkage of electron wave function at high fields. Magnetoresistance shows a $\mathrm{B}^{2}$ dependence at low fields and is temperature dependent, whereas at high fields it is independent of temperature and exhibits a $\mathrm{B}^{1 / 2}$ dependence [11].

The thermal stability of any system depends on the host structure and the amount of intercalated species [12]. It also depends on how well the guest species are bonded to the host atoms. It is known that the stability decreases with improving crystallinity of the host system, since, with increasing crystallinity, not only intercalation but also deintercalation seems easier.

In this work, we examine the changes in the structural and mechanical properties of the a-C samples due to iodine incorporation by X-ray photoelectron spectroscopy (XPS) and microhardness measurements respectively. Electrical resistivity and magnetoresistance measurements performed on the a-C: I samples are discussed. Thermal stability of these a-C: I samples are analyzed by Thermogravimetric (TG) and derivative TG (DTG) analysis. 


\section{Experimental details}

The a-C: I films were prepared by vapor phase pyrolysis using home-built horizontal double zone furnace set-up. Carbon rich organic precursor, maleic anhydride was used as starting material and iodine crystals as an intercalation agent. Both the materials were taken in different weight ratios and were pyrolysed at various temperatures $\left(700-980^{\circ} \mathrm{C}\right)$. The a-C: I films were deposited on unpolished quartz substrates. Detailed description of sample preparation is given elsewhere [7]. Samples prepared are conducting and contamination free. The degree of iodination, $I / P$ represents the initial weight ratio of iodine to maleic anhydride.

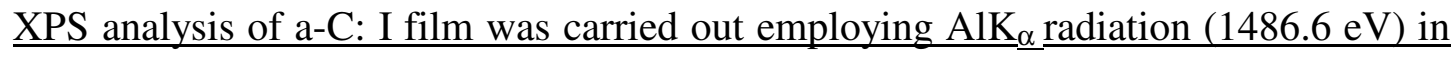
an ESCA-3 Mark II Spectrometer (VG Scientific Ltd., England) at a pass energy of 50 $\underline{\mathrm{eV}}$. The XPS analysis was performed at a base pressure of $10^{-9}$ Torr in the analyzer chamber. The binding energies were measured with a precision of $\pm 0.2 \mathrm{eV}$. In order to avoid the specimen charging, the film was mounted on the sample probe using silver paste. As the a-C: I sample was highly conducting, no charging effect was observed during the measurement.

Microhardness tests were conducted (HMV-2000 Shimadzu) on a-C and a-C: I films (film thickness of the range $1-2 \mu \mathrm{m}$ ) deposited on polished quartz substrates by microindentation technique. 
DC electrical conductivity and transverse magnetoresistance measurements were performed on these samples in a liquid helium cryostat (Janis Supervaritemp Cryostat) from $300 \mathrm{~K}$ down to $1.3 \mathrm{~K}$ with a magnetic field of $0-7 \mathrm{~T}$, which was obtained by using superconducting magnet in the cryostat. The measurements were carried out by using a standard four-probe technique. The sample contacts were made using fine enamelled copper wires as contact leads and silver paste as contact material. The power dissipated into the sample is less than $1 \mu \mathrm{W}$ at low temperatures in order to avoid the heat-induced damage of the sample.

Thermal stability of a-C and a-C: I (I/P) samples was analyzed using a PYRIS EXSTAR-6000 thermal analyzer. The samples were heated from 25 to $600^{\circ} \mathrm{C}$ at a rate of $10^{\circ} \mathrm{C} / \mathrm{min}$ in the presence of $\mathrm{N}_{2}$ gas atmosphere with a flow rate of $150 \mathrm{ml} / \mathrm{min}$.

\section{Results and discussion}

\subsection{Sample characterization by XPS and Microhardness test}

$\underline{\mathrm{XP} \text { spectra of a-C: } \mathrm{I}(I / P=1) \text { film deposited at } 900^{\circ} \mathrm{C} \text { on unpolished quartz substrate }}$

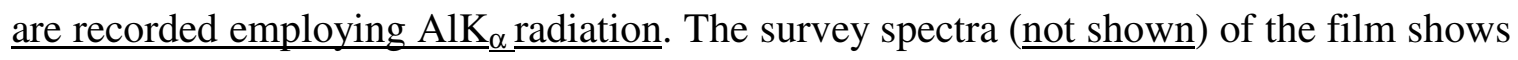
the presence of iodine together with nitrogen, oxygen and also the expected carbon in the intercalated film. Nitrogen and oxygen atoms may be incorporated into the sample during the pyrolysis process. Fig. 1 shows I(3d) core level region of the film. Accordingly, $\mathrm{I}\left(3 \mathrm{~d}_{5 / 2,3 / 2}\right)$ peaks at 620 and $631 \mathrm{eV}$ respectively can be attributed to iodine atom in the sample. Thus XPS analysis infers that iodine atoms get incorporated via other atoms (probably carbon atoms) and not as pure iodine molecule [13]. 
Powder X-ray diffraction (XRD) pattern of a-C:I samples show crystalline peaks with an amorphous background, which confirms that the system has two phases, amorphous and crystalline. The crystalline peaks correspond to the carbon-iodine system. The surface morphological changes in the a-C:I films are studied by Scanning electron microscopy. The increase in iodine concentration accounts for the highly localized and uniform cluster pattern in the film [7].

The microhardness test is conducted on the a-C and a-C: I film. The hardness value of the film grown on polished quartz substrate, is found by applying a determined load (100$200 \mathrm{gm}$ ) on the sample and then measuring the diamond indenter and diagonally received pattern. The comparative plot of the microhardness of the a-C $(I / P=0)$ and a-C: I

$(I / P=0.25$ and $I / P=1)$ samples are as shown in Fig. 2. The studies account for the decrease in the value of microhardness with the increase in the iodine concentration. Earlier work [7] shows that the iodine incorporation into the a-C samples accounts for a uniform and dense clustering of carbon atoms. Robertson and O'Reilly [14] showed that, in a-C a mixture of $s p^{2}$ and $s p^{3}$ carbon sites will tend to segregate into $s p^{2}$-bonded graphitic clusters embedded in a $s p^{3}$-bonded matrix. The $s p^{2}$ clusters were found to control the electronic properties and the connectivity of the $s p^{3}$ matrix largely controls the mechanical properties. The graphitic clustering of $s p^{2}$ sites reduces the number of useful constraints in a network. Hence $s p^{2}$ sites and clustering can severely lower the hardness of a-C samples [15]. 


\subsection{Electrical conductivity}

The conductivity of iodinated amorphous conducting carbon films depends on the pyrolysis temperature and iodine concentration [7]. The resistivity ratio, $\rho_{r}=\rho(1.3 \mathrm{~K}) / \rho(300 \mathrm{~K})$ defines the measure of disorder in the a-C system [16]. As the

disorder in the system increases, $\rho_{r}$ increases. Variation of normalized resistivity and room temperature conductivity as a function of pyrolysis temperature for a-C: I sample with $I / P=1$ prepared at $900^{\circ} \mathrm{C}$ is as shown in Fig. 3. Inset is the sample showing a

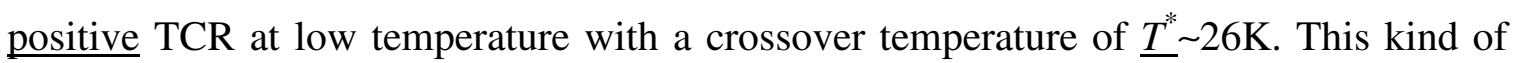
behavior was previously observed in PAN-based fiber [17] and pyrocarbon [18]. From the plot it is evident that the a-C:I samples are less disordered and more conducting than the a-C samples. This infers that the iodine incorporation into the a-C system induces a local ordering. Also iodine intercalation brings about charge transfer, resulting in the enhancement of the density of states, thereby increasing the conductivity of the sample. Appreciable change in $\rho_{r}$ is observed for samples in the insulating and critical regime than for metallic samples. Previous report on the heat-treated hard and soft carbon [4] samples showed that the temperature dependence of resistivity, $\rho_{r}$ decreases with acceptor doping. The overall conductivity of the sample may be understood as due to the tunneling of charge carriers between the remote conductive chains $[9, \underline{19}]$. 


\subsection{Effect of magnetic field}

The variation of magnetoresistance, given by $M R=\left[\left(R_{B}-R_{0}\right) / R_{0}\right]$ as a function of magnetic field in iodinated a-C samples prepared at $900^{\circ} \mathrm{C}$ with different $I / P$ values is shown in Fig. 4. From the plot, it is evident that the magnetoresistance exhibits different behavior at different temperatures. The value of magnetoresistance decreases appreciably with the iodine incorporation into the a-C samples for temperatures greater than $1.3 \mathrm{~K}$. At low temperatures $(\leq 6 \mathrm{~K})$, the magnetoresistance is high and positive, whereas it is low and negative at measurement temperatures $\geq 8 \mathrm{~K}$. For low temperature measurements, magnetoresistance tends to saturate at high fields. The large change in the magnetoresistance is observed at high fields and low temperatures compared to the relatively small changes around low fields and high temperatures $\geq 8 \mathrm{~K}$. The behavior of magnetoresistance at high fields is very much influenced by the iodine incorporation. But in saturation intercalated samples $(I / P=1.14)$ at high fields, magnetoresistance becomes positive for temperatures till $20 \mathrm{~K}$. This is due to the ionized impurity scattering introduced by the iodine incorporation, which also depends upon the iodine concentration. Iodine intercalation suppresses the influence of structural disorder similar to the effect of heat treatment and hence diminish the inelastic scattering in the presence of magnetic field. 


\subsection{Thermal analysis}

Thermal stability of any intercalated system depends on the bonding between the host system and the guest species. XRD studies on a-C: I sample showed crystalline peaks corresponding to the carbon-iodine system [7], but the bonding between carbon and iodine may be weak. The intercalated iodine will be present on the defects and discontinuities of the host structure. When these samples are subjected to heating, adsorbed iodine can easily come out of the structure, as there are no strong bonds between the trapped iodine and the carbon atoms.

Thermogravimetric analysis is carried out on a-C: I samples with $I / P=0$ and $I / P=1$ prepared at $900^{\circ} \mathrm{C}$. The TG and DTG curves (Fig. 5(a) \& 5(b)) show gradual weight loss, indicating no sharp threshold temperature for iodine desorption. The results will be discussed in three temperature ranges, with respect to the appreciable changes taking place in these regions. In the temperature range $27-100^{\circ} \mathrm{C}$, weight loss of $8 \%$ is observed in a-C sample at around $80^{\circ} \mathrm{C}$ (Fig. 5(a)), which can be attributed to a removal of moisture content from the sample. A sharp peak at this temperature in the DTG curve confirms the weight loss. Even in a-C: I $(I / P=1)$ sample (Fig. $5 b)$, weight loss of $8 \%$ at $75^{\circ} \mathrm{C}$ corresponds to removal of moisture, which is also witnessed by a sharp peak in the

DTG curve. In this temperature range, small amount of intercalated iodine, which is loosely bound to carbon atoms, may also come out of the system. TG curve of a-C sample shows a weight loss of $7 \%$ in the temperature range $100-200^{\circ} \mathrm{C}$, whereas for 
a-C: I sample it accounts for a weight loss of $27 \%$, which can be attributed to the deintercalation of iodine, shown by a very sharp peak (DTG curve) at around $200^{\circ} \mathrm{C}$. $\underline{\mathrm{A}}$ gradual weight loss is observed for both a-C and a-C: I samples in the high temperature $\underline{\text { range, which may be due to the decomposition of carbon to form } \mathrm{CO} \text { and } \mathrm{CO}_{2}}$. A TG and DTG curve for both samples in this temperature range seems to be same, which confirms that around $200^{\circ} \mathrm{C}$ most of the intercalated iodine is leaving the a-C: I sample. The remaining iodine, which may be bonded to carbon atoms or may be trapped in the defects, gets deintercalated above $200^{\circ} \mathrm{C}$. Powder XRD pattern of a-C: I sample, heat treated at $300^{\circ} \mathrm{C}$ shows no crystalline peaks corresponding to carbon-iodine system, which thereby confirms the release of iodine from the a-C: I sample by thermal treatment. Thermal analysis of fluorine-intercalated carbon fibres [12] also shows a change in crystallinity of intercalated fibers during decomposition.

\section{Conclusions}

We have studied the effect of iodination on the structural and transport properties of a-C films prepared by vapor phase pyrolysis. XP spectra of a-C:I film shows $\mathrm{I}\left(3 \mathrm{~d}_{5 / 2,3 / 2}\right)$ peaks at 620 and $631 \mathrm{eV}$ respectively, which corresponds to iodine in the sample. Iodine incorporation accounts for the decrease in the microhardness of the a-C samples due to increase in clustering of carbon atoms. Appreciable increase in the conductivity and decrease in $\rho_{r}$ is observed for iodine intercalated samples. The value of magnetoresistance is found to decrease with the increase in the iodine concentration at temperatures higher 
than $1.3 \mathrm{~K}$. Thermal stability of the a-C: I samples are studied by TG and DTG analysis, which confirms the deintercalation of iodine by heat treatment.

\section{Acknowledgements}

We are grateful to DST, CSIR and STC for financial support. We thank Prof. M. S. Hegde of Solid State and Structural Chemistry Unit, IISc, for providing XPS facility. 


\section{References}

[1] P.A. Lee, T.V. Ramakrishnan, Rev. Mod. Phys. 57 (1985) 287.

[2] B.I. Shklovskii, A.L. Efros, Electronic properties of Doped Semiconductors, Springer-Verlag, Berlin, 1984.

[3] E. Medina, M. Kardar, Phys. Rev. B 46 (1992) 9984.

[4] Y. Komatsu, Carbon 7 (1969) 177.

[5] F. Carmona, P. Delhaes, G. Keryer and J. P. Manceau, Solid State Comm. 14 (1974) 1183.

[6] N. F. Mott, E. A. Davis, Electronic processes in noncrystalline materials, Oxford University Press, Oxford, 1979.

[7] Latha Kumari, V. Prasad, S. V. Subramanyam, Carbon 41 (2003) 1841.

[8] L. Grigorian, K. A. Williams, S. Fang, G. U. Sumanasekara, A. L. Loper, E. C. Dickey, S. J. Pennycook, P. C. Eklund, Phys. Rev. Lett. 80 (1998) 5560.

[9] S. Tanuma, in: S. Yoshimura, R. P. H. Chang (Eds.), Super Carbon: Synthesis, Properties and Applications, Springer-Verlag, Germany, 1998, p. 132.

[10] M. Allon-Alaluf, N. Croitoru, Appl. Phys. Lett. 69 (1996) 2932.

[11] J. S. Dugdale, The Electrical Properties of Disordered Metals, Cambridge University Press, Cambridge, 1995.

[12] V. Gupta, R. B. Mathur, O. P. Bahl, A. Tressaud, S. Flandrois, Synth. Met. 73 (1995) 69.

[13] M. Allon-Alaluf, L. Klibanov, N. Croitoru, Diamond Relat. Mater. 5 (1996) 1497.

[14] J. Robertson, E. P. O’Reilly, Phys. Rev. B 35 (1987) 2946.

[15] J. Robertson, Phys. Rev. Lett. 68 (1992) 220.

[16] M. Reghu, in: H. S. Nalwa (Ed.), Handbook of organic conductive molecules and polymers, John Wiley \& Sons Ltd., West Sussex, 1997, p. 47.

[17] I. L. Spain, K. J. Volin, H. A. Goldberg, I. L. Kalnin, Solid State Comm. 45 (1983) 817. 
[18] A. Faißt, Hv. Löhneysen, Carbon 40 (2002) 321.

[19] A. Sayeed, Ph.D. Thesis, Indian Institute of Science, India, 1998. 
Fig.1. XP spectra of I (3d) core level region in the a-C: I $(I / P=1)$ film prepared at $900^{\circ} \mathrm{C}$.

Fig.2. Microhardness of a-C and a-C: $\mathrm{I}(I / P=0.25$ and $I / P=1)$ films prepared at $900^{\circ} \mathrm{C}$.

Fig.3. Variation of normalized resistivity and conductivity as a function of pyrolysis temperature for a-C: I $(I / P=1)$ films. Inset: sample showing a positive TCR below $T^{*} \sim 26 \mathrm{~K}$

Fig.4. Magnetoresistance as a function of magnetic field at various temperatures for a samples with (a) $I / P=0$, (b) $I / P=0.38$, (c) $I / P=0.5$ and (d) $I / P=1.14$ prepared at $\underline{900^{\circ} \mathrm{C}}$.

Fig.5. TG-DTG curves of the (a) a-C and (b) a-C: $\mathrm{I}(I / P=1)$ samples prepared at $900^{\circ} \mathrm{C}$. 


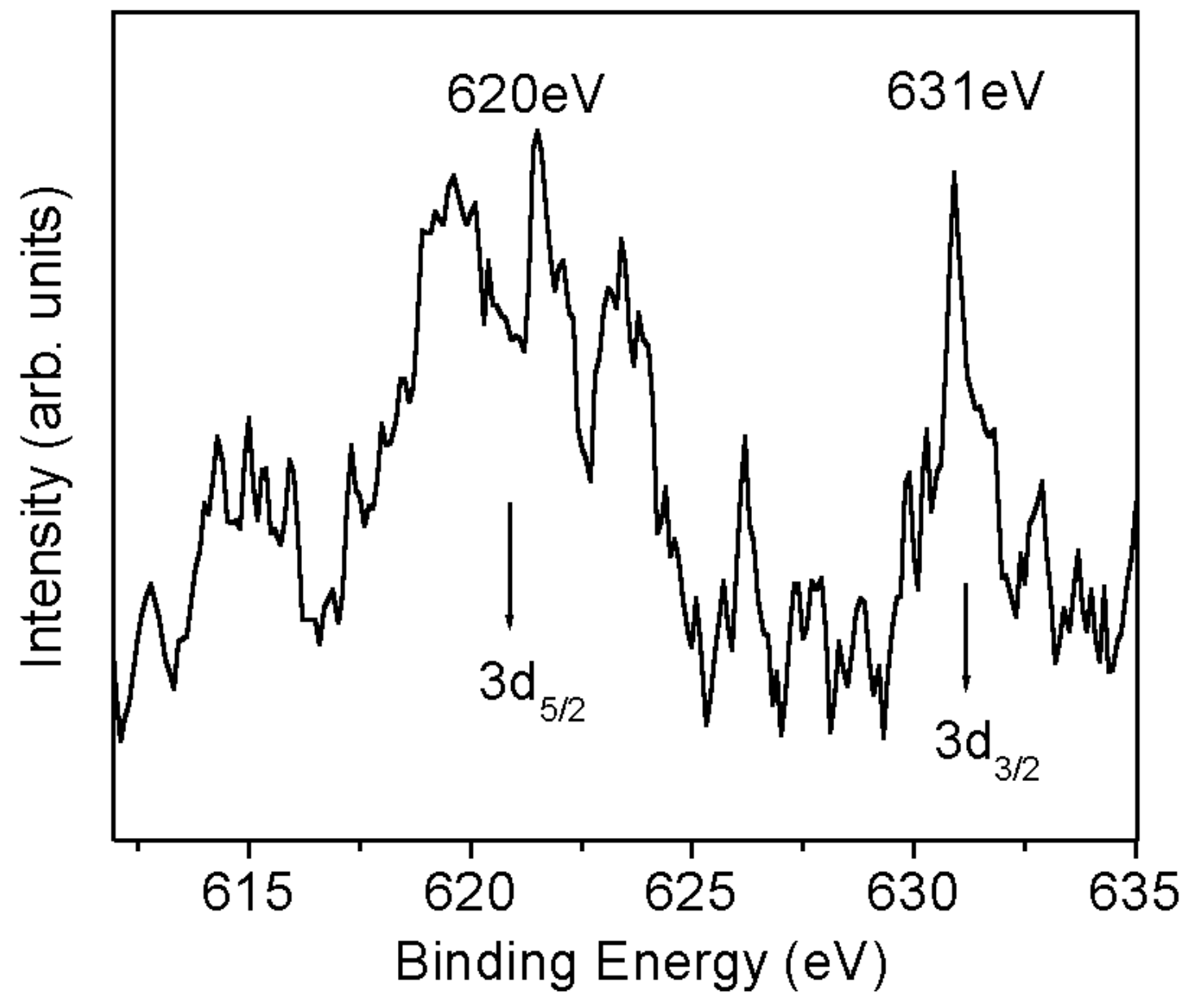

Fig.1.

Latha Kumari et al. 


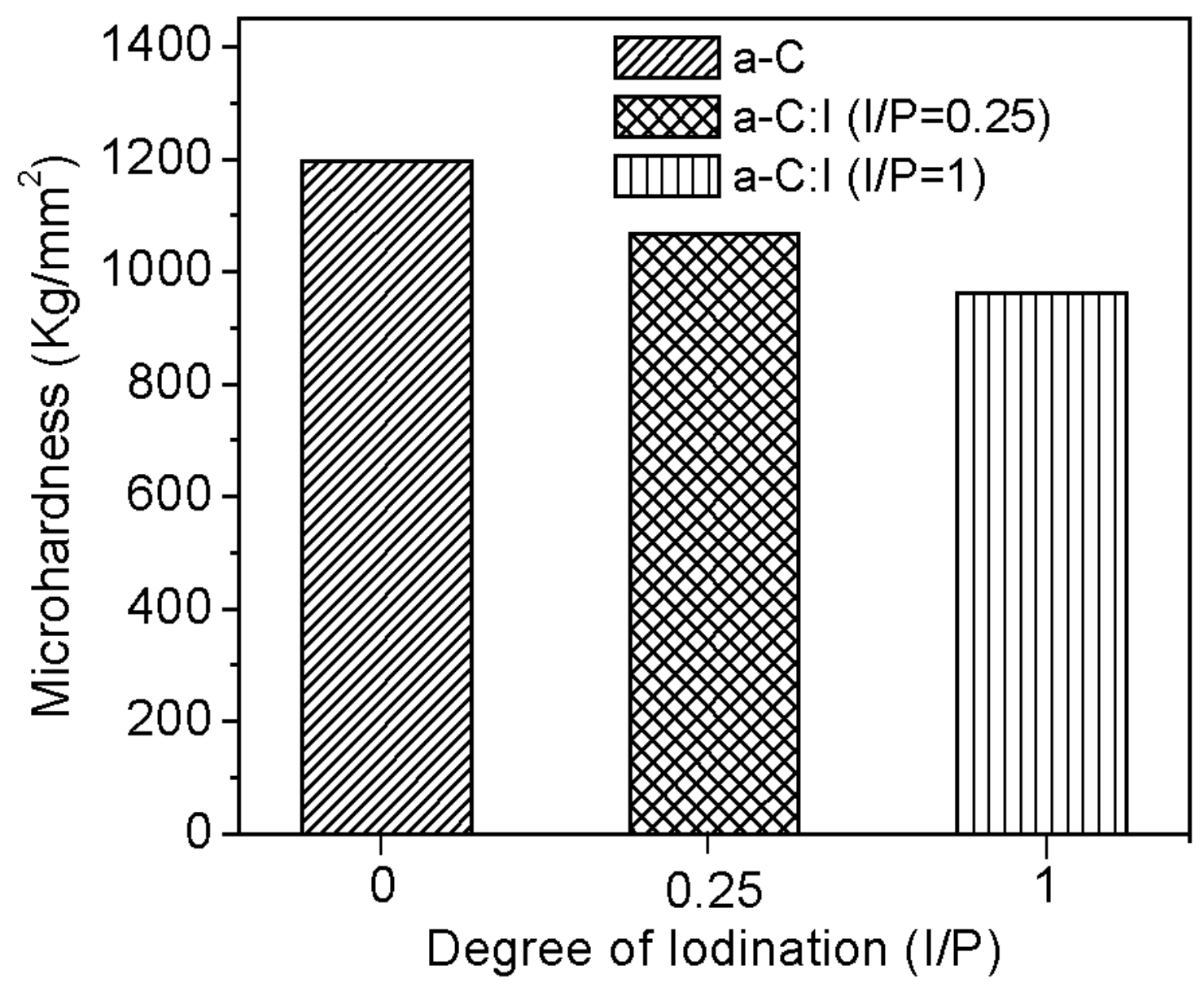

Fig.2.

Latha Kumari et al. 


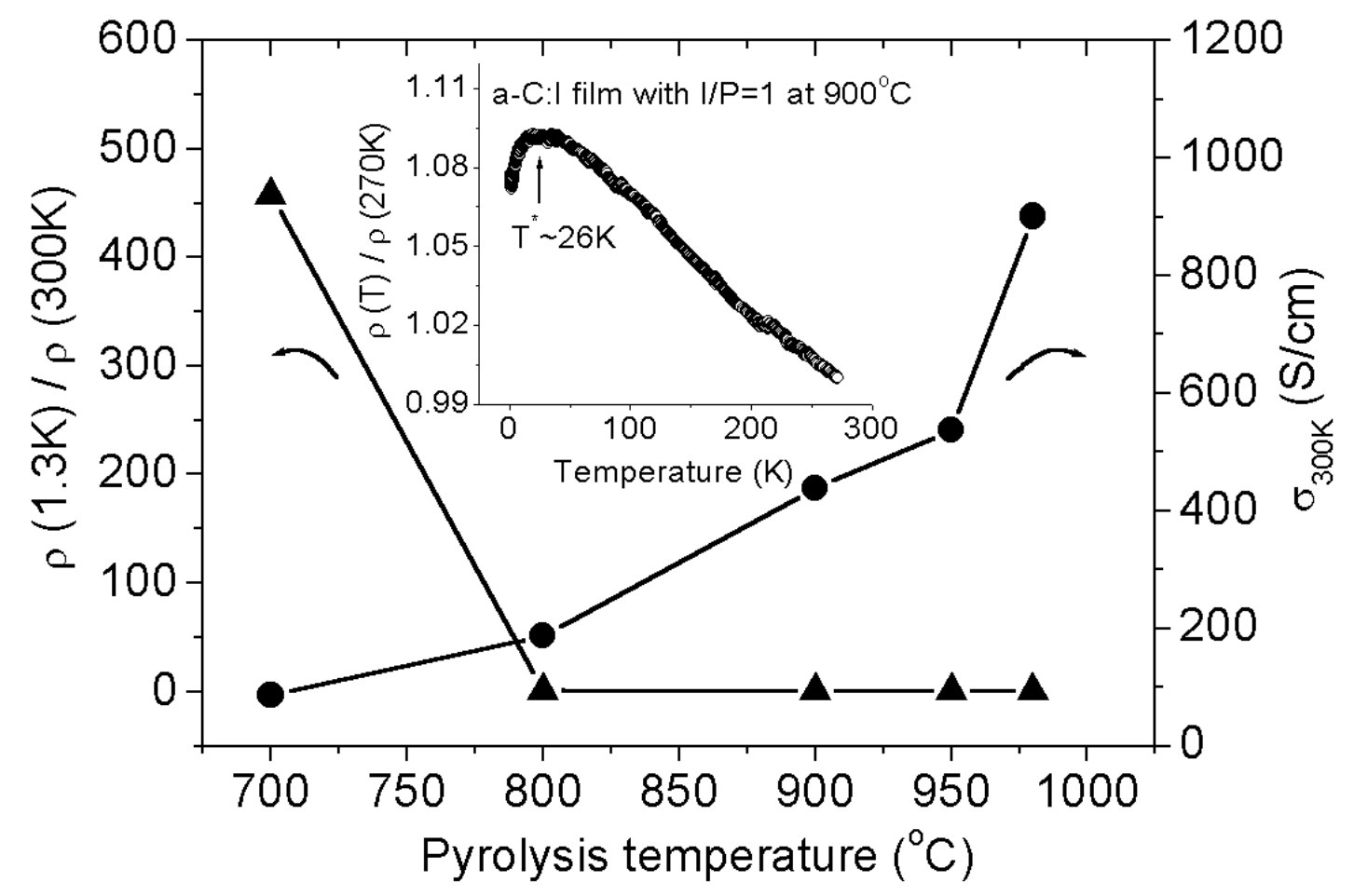

Fig.3.

Latha Kumari et al. 


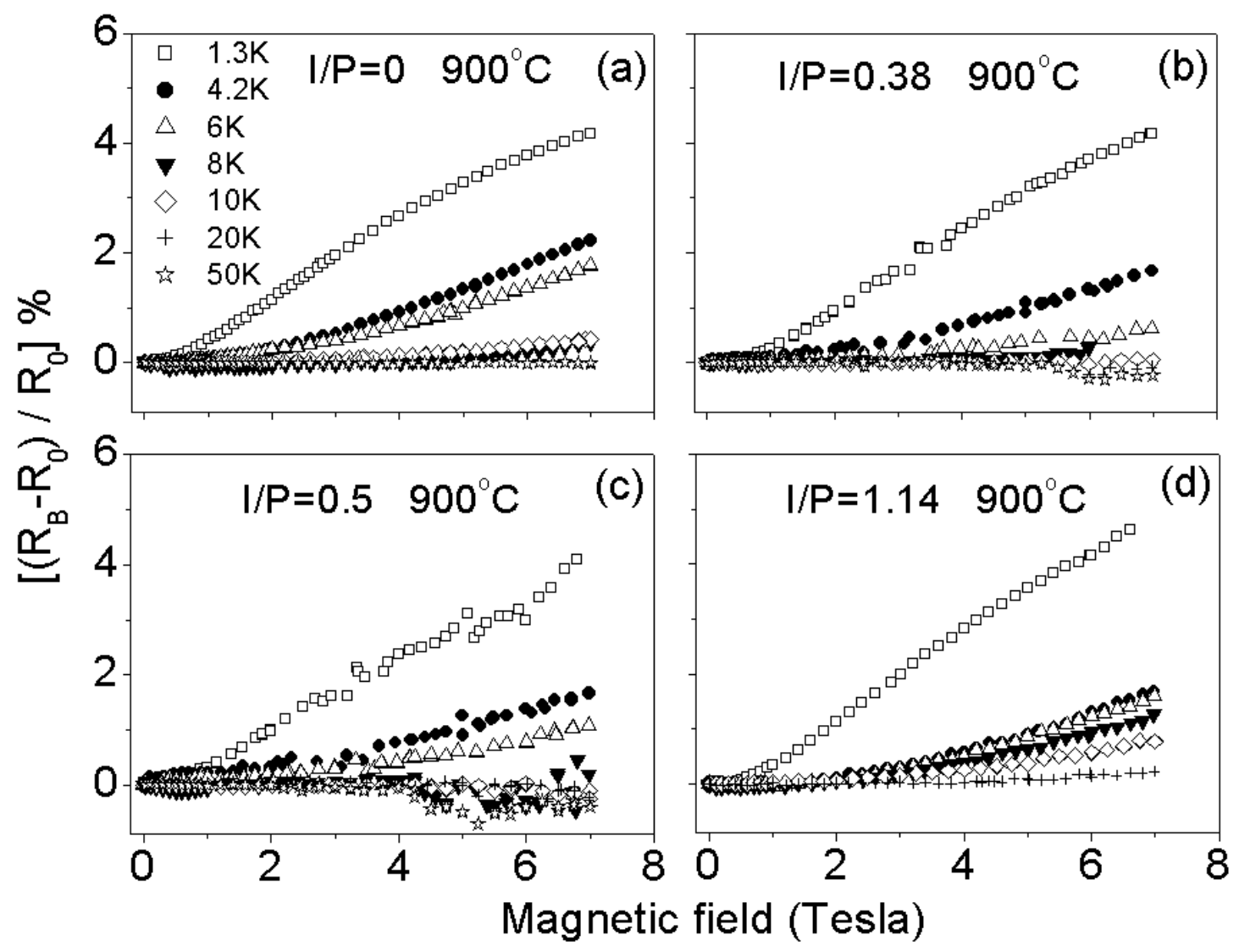

Fig.4.

Latha Kumari et al. 


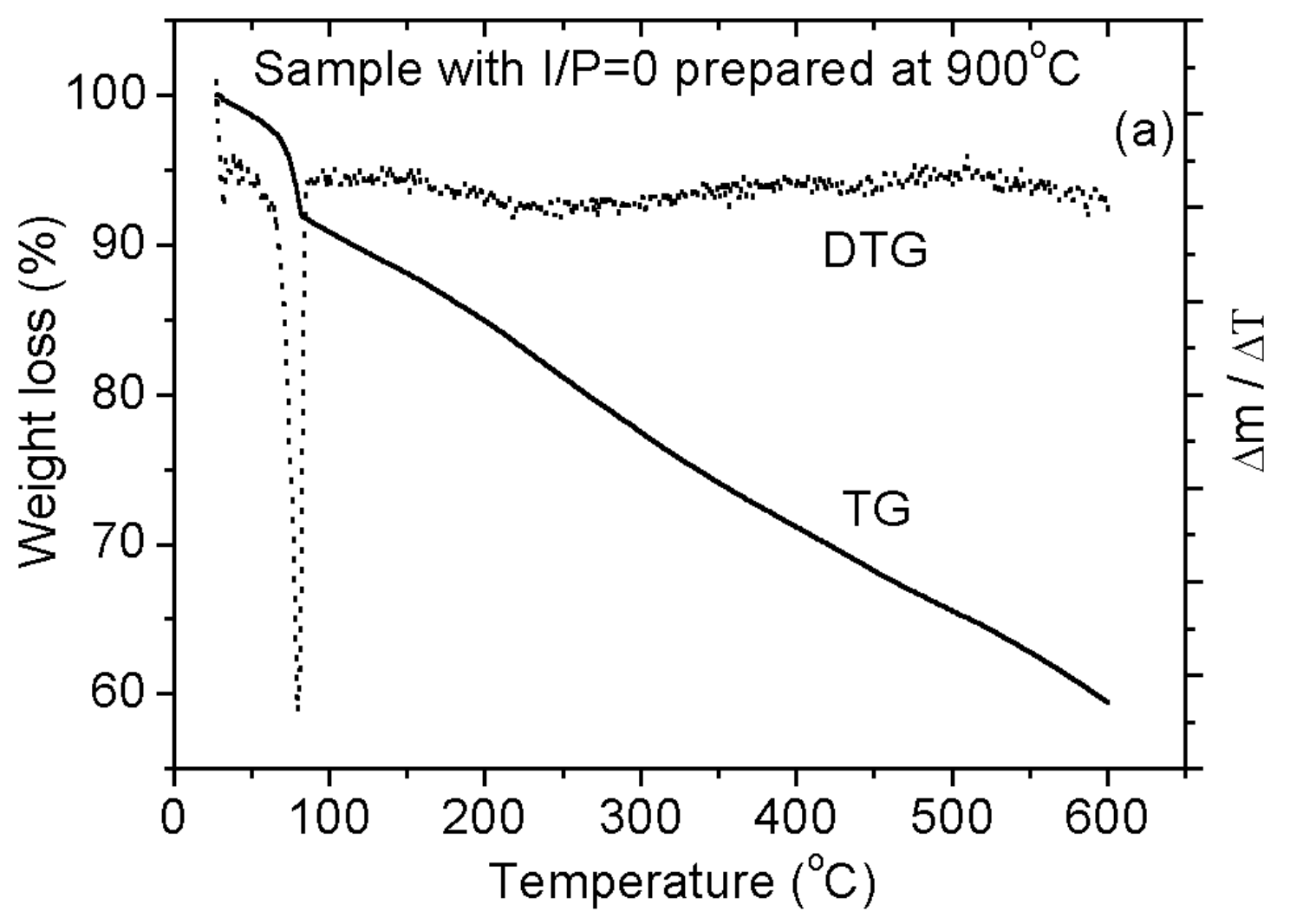

Fig.5a.

Latha Kumari et al. 


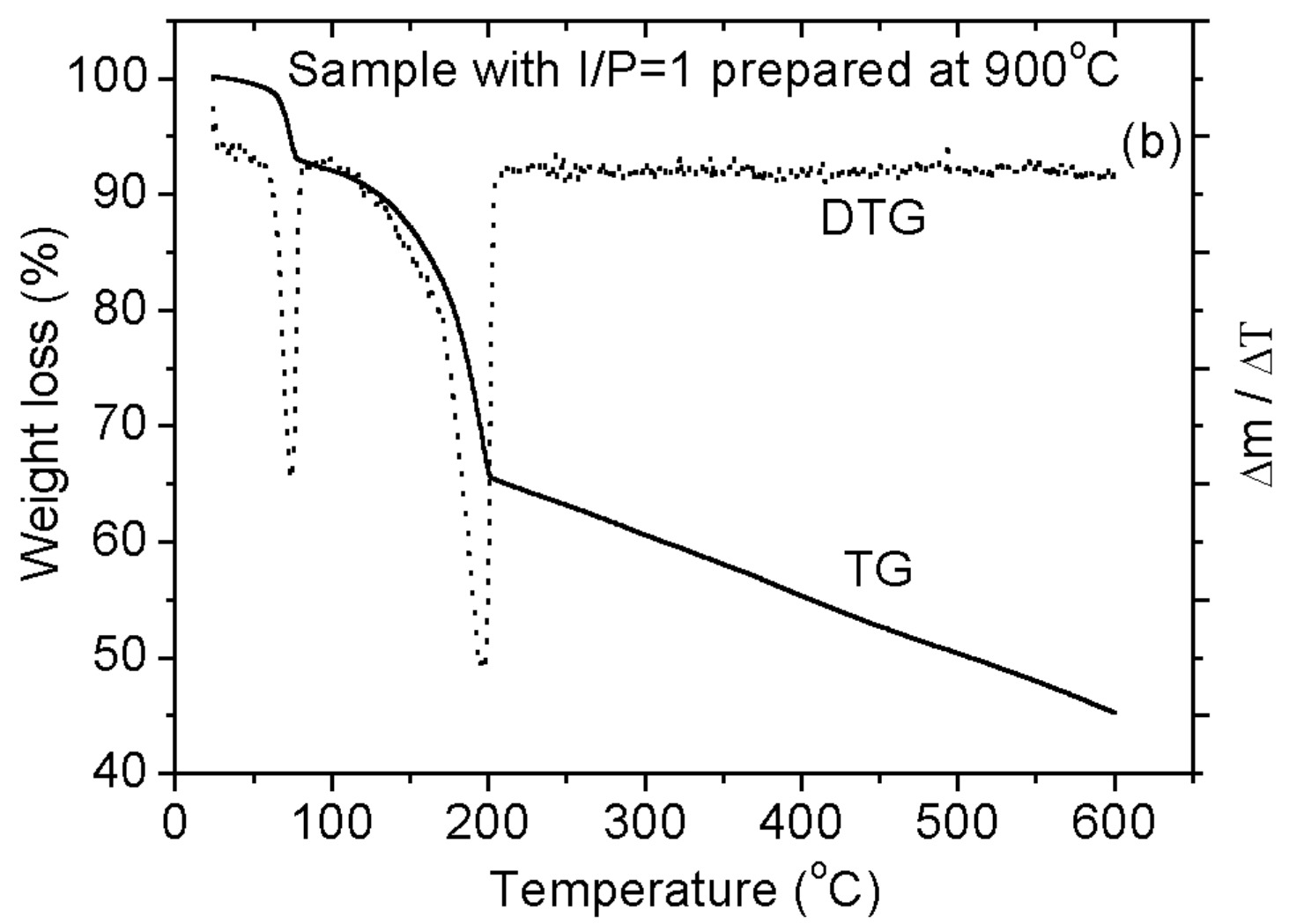

Fig.5b.

Latha Kumari et al. 